\title{
One million structures and counting
}

\author{
Suzanna Ward ${ }^{a}$ and Amy Sarjeant ${ }^{b}$ \\ aThe Cambridge Crystallographic Data Centre, 12 Union Road, Cambridge, CB2 1EZ, UK, \\ ward@ccdc.cam.ac.uk \\ ${ }^{\mathrm{b}}$ The Cambridge Crystallographic Data Centre, 252 Nassau St. Princeton NJ 08542, USA, \\ sarjeant@ccdc.cam.ac.uk
}

This year the world of structural chemistry will reach a tremendous milestone - the publication of over one million organic and metal organic crystal structures. Each one of these 1 million structures is shared through the Cambridge Structural Database (CSD) and is something the entire scientific community and the ACA community can be proud of. It is thanks to this community's exemplary data sharing practices, as well as the thoughts of visionaries in the field, that this collection of data exists.

Today the CSD and the accompanying software are used by scientists worldwide working in over 70 countries, and the knowledge derived from the structural data contained in the CSD has underpinned fundamental chemical discoveries and played a key role in designing new materials from drugs to pigments and beyond.

This talk will look at some of the research that the CSD has enabled and highlight the knowledge and insights that have been derived from this collection of data. We will finish by celebrating and thanking a few of the many heroes of the Cambridge Structural Database who have contributed their data to this amazing resource. 